\title{
2006 FISH CONSUMPTION ADVISORIES
}

NOTE: 2006 Advisories marked in Bold are New or Revised

\section{STATEWIDE FISH CONSUMPTION ADVISORIES}

STATEWIDE ESTUARINE \& MARINE WATERS

(All coastal waters except those under Waterbody Specific Advisories)

\begin{tabular}{|c|c|c|}
\hline & GENERAL POPULATION & HIGH-RISK INDIVIDUAL ${ }^{(1)}$ \\
\hline SPECIES & EAT NO MORE THAN: $(2,3)$ & EAT NO MORE THAN: ${ }^{(2,3)}$ \\
\hline STRIPED BASS & One meal per month & \multirow{4}{*}{ Do Not Eat } \\
\hline $\begin{array}{l}\text { BLUEFISH } \\
\text { (greater than } 6 \mathrm{lbs} / 24 \text { inches) }\end{array}$ & Four meals per year & \\
\hline $\begin{array}{l}\text { BLUEFISH } \\
\text { (less than 6lbs/24 inches) }\end{array}$ & One meal per month & \\
\hline AMERICAN EEL & Four meals per year & \\
\hline AMERICAN LOBSTER & \multicolumn{2}{|c|}{ Do Not Eat the Green Gland (a.k.a., Tomalley or Hepatopancreas) } \\
\hline \multicolumn{3}{|c|}{$\begin{array}{l}\text { GENERAL FRESHWATER ADVISORIES: } \\
\text { (For all freshwater fish species \& waterbodies not covered by a consumption advisory) } \\
\text { General Population - Eat No More Than One Meal Per Week } \\
\text { High-risk Individual - Eat No More Than One Meal Per Month }\end{array}$} \\
\hline \multicolumn{3}{|c|}{$\begin{array}{l}\text { STATEWIDE FRESHWATERS } \\
\text { (All freshwaters except PINELANDS REGION and those listed in Waterbody Specific Advisories) }\end{array}$} \\
\hline & GENERAL POPULATION & HIGH-RISK INDIVIDUAL \\
\hline SPECIES & EAT NO MORE THAN: & EAT NO MORE THAN: \\
\hline LARGEMOUTH BASS & \multirow{3}{*}{ One meal per week } & \multirow{5}{*}{ One meal per month } \\
\hline SMALLMOUTH BASS & & \\
\hline CHAIN PICKEREL & & \\
\hline YELLOW BULLHEAD & \multirow{3}{*}{ No restrictions } & \\
\hline SUNFISH $^{(4)}$ & & \\
\hline BROWN BULLHEAD & & One meal per week \\
\hline
\end{tabular}

NOTE: To reduce your exposure, eat those fish with the lowest meal restrictions. Do not combine meal restrictions. (For example: If you eat a fish at "one meal per week", then do not eat a different fish at "four meals per year"). 


\section{REGIONAL FRESHWATER ADVISORIES PINELANDS REGION}

(All waters of the Pinelands Region except Waterbody Specific Advisories listed with a "P" notation)

\begin{tabular}{||l||c||c||}
\hline \hline LARGEMOUTH BASS & One meal per month & Do not eat \\
\hline CHAIN PICKEREL & & \\
\hline BROWN BULLHEAD & One meal per week & One meal per month \\
\hline YELLOW BULLHEAD & & OnNFISH \\
\hline SU $^{(4)}$ &
\end{tabular}

\section{WATERBODY SPECIFIC FISH CONSUMPTION ADVISORIES}

\begin{tabular}{|c|c|c|c|}
\hline \multicolumn{4}{|c|}{ ESTUARINE \& MARINE WATERS } \\
\hline \multirow[b]{2}{*}{ WATERBODY } & \multirow[b]{2}{*}{ SPECIES } & $\begin{array}{c}\text { GENERAL } \\
\text { POPULATION }\end{array}$ & $\begin{array}{c}\text { HIGH-RISK } \\
\text { INDIVIDUALS }\end{array}$ \\
\hline & & $\begin{array}{c}\text { EAT NO MORE } \\
\text { THAN: }\end{array}$ & $\begin{array}{c}\text { EAT NO MORE } \\
\text { THAN: }\end{array}$ \\
\hline \multirow{5}{*}{$\begin{array}{l}\text { NEWARK BAY COMPLEX } \\
\text { Including Newark Bay, tidal } \\
\text { Hackensack River, Arthur } \\
\text { Kill, Kill Van Kull and tidal } \\
\text { tributaries. }\end{array}$} & Blue Crab* & \multicolumn{2}{|c|}{ Do not harvest, ${ }^{(5)}$ Do not eat } \\
\hline & Striped Bass* & Four meals per year & \multirow{4}{*}{ Do not eat } \\
\hline & American Eel* & \multirow{2}{*}{ Do not eat } & \\
\hline & White Perch & & \\
\hline & White Catfish & One meal per year & \\
\hline \multirow{2}{*}{$\begin{array}{l}\text { TIDAL PASSAIC RIVER } \\
\text { Dundee Dam to Newark Bay } \\
\text { and tributaries. }\end{array}$} & All Fish \& Shellfish* & \multicolumn{2}{|c|}{ Do not eat } \\
\hline & Blue Crab* & \multicolumn{2}{|c|}{ Do not harvest, ${ }^{(5)}$ Do not eat } \\
\hline \multirow{6}{*}{$\begin{array}{l}\text { HUDSON RIVER } \\
\text { Downstream of NY-NJ } \\
\text { border including the Upper } \\
\text { New York Bay }\end{array}$} & Striped Bass* & Four meals per year & \multirow{3}{*}{ Do not eat } \\
\hline & American Eel* & \multirow{2}{*}{ One meal per year } & \\
\hline & White Perch & & \\
\hline & Winter Flounder & \multicolumn{2}{|c|}{ One meal per month } \\
\hline & White Catfish & \multicolumn{2}{|c|}{ Do not eat } \\
\hline & Blue Crab & \multicolumn{2}{|c|}{$\begin{array}{c}\text { One meal of } 7 \text { crabs per week } \\
\text { Do not eat green gland (hepatopancreas); } \\
\text { Discard cooking liquid } \\
\end{array}$} \\
\hline
\end{tabular}

NOTE: To reduce your exposure, eat those fish with the lowest meal restrictions. Do not combine meal restrictions. (For example: If you eat a fish at "one meal per week", then do not eat a different fish at "four meals per year"). 


\section{WATERBODY SPECIFIC FISH CONSUMPTION ADVISORIES}

\begin{tabular}{|c|c|c|c|}
\hline \multicolumn{4}{|c|}{ ESTUARINE \& MARINE WATERS } \\
\hline \multirow[b]{2}{*}{ WATERBODY } & \multirow[b]{2}{*}{ SPECIES } & $\begin{array}{l}\text { GENERAL } \\
\text { POPULATION }\end{array}$ & $\begin{array}{l}\text { HIGH-RISK } \\
\text { INDIVIDUALS }\end{array}$ \\
\hline & & $\begin{array}{l}\text { EAT NO MORE } \\
\text { THAN: }\end{array}$ & $\begin{array}{l}\text { EAT NO MORE } \\
\text { THAN: }\end{array}$ \\
\hline \multirow{9}{*}{$\begin{array}{l}\text { RARITAN BAY } \\
\text { COMPLEX } \\
\text { Includes the Raritan Bay, } \\
\text { tidal Raritan River (to Rte. } 1 \\
\text { bridge) and the tidal portions } \\
\text { of all tributaries. }\end{array}$} & American Lobster & \multicolumn{2}{|c|}{$\begin{array}{l}\text { One meal per week } \\
\text { Do not eat green gland (hepatopancreas) } \\
\text { Discard cooking liquid }\end{array}$} \\
\hline & Weakfish & \multirow{2}{*}{ One meal per month } & \multirow{2}{*}{ Do not eat } \\
\hline & Striped Bass & & \\
\hline & White Perch & One meal per year & Do not eat \\
\hline & Winter Flounder & \multirow{2}{*}{\multicolumn{2}{|c|}{ One meal per month }} \\
\hline & Porgy & & \\
\hline & American Eel & One meal per year & \multirow{2}{*}{ Do not eat } \\
\hline & White Catfish & Four meals per year & \\
\hline & Blue Crab & \multicolumn{2}{|c|}{$\begin{array}{l}\text { One meal of } 7 \text { crabs per month } \\
\text { Do not eat green gland (hepatopancreas); } \\
\text { Discard cooking liquid }\end{array}$} \\
\hline $\begin{array}{l}\text { COASTAL TRIBUTARIES } \\
\text { Navesink River, Shrewsbury } \\
\text { River, Shark River, Toms } \\
\text { River \& Mullica River. }\end{array}$ & American Eel & One meal per month & One meal per month \\
\hline \multirow{7}{*}{$\begin{array}{l}\text { LOWER (TIDAL) } \\
\frac{\text { DELAWARE RIVER }}{\text { Trenton, NJ to PA/DE line, }} \\
\text { including all tributaries to the } \\
\text { head of tide. }\end{array}$} & Largemouth Bass & \multirow{2}{*}{ No restrictions } & \multirow{2}{*}{ One meal per week } \\
\hline & Hybrid Striped Bass & & \\
\hline & American eel & \multirow{2}{*}{ One meal per year } & \multirow{5}{*}{ Do not eat } \\
\hline & Channel Catfish & & \\
\hline & White Catfish & One meal per month & \\
\hline & Striped Bass & Four meals per year & \\
\hline & White Perch & Four meals per year & \\
\hline $\begin{array}{l}\text { DELAWARE RIVER } \\
\text { ESTUARY- DE/NJ/PA } \\
\text { border to C\&D Canal }\end{array}$ & All finfish & \multicolumn{2}{|c|}{ Do not eat } \\
\hline
\end{tabular}

NOTE: To reduce your exposure, eat those fish with the lowest meal restrictions. Do not combine meal restrictions. (For example: If you eat a fish at "one meal per week", then do not eat a different fish at "four meals per year"). 


\section{WATERBODY SPECIFIC FISH CONSUMPTION ADVISORIES}

\begin{tabular}{|c|c|c|c|}
\hline \multicolumn{4}{|c|}{ ESTUARINE \& MARINE WATERS } \\
\hline \multirow[b]{2}{*}{ WATERBODY } & \multirow[b]{2}{*}{ SPECIES } & $\begin{array}{l}\text { GENERAL } \\
\text { POPULATION }\end{array}$ & $\begin{array}{l}\text { HIGH-RISK } \\
\text { INDIVIDUALS }\end{array}$ \\
\hline & & $\begin{array}{l}\text { EAT NO MORE } \\
\text { THAN: }\end{array}$ & $\begin{array}{l}\text { EAT NO MORE } \\
\text { THAN: }\end{array}$ \\
\hline \multirow{8}{*}{$\begin{array}{l}\text { DELAWARE ESTUARY \& } \\
\text { BAY } \\
\text { C\&D Canal to the mouth of } \\
\text { Delaware Bay }\end{array}$} & $\begin{array}{l}\text { Bluefish (less than } \\
14 \text { inches) }\end{array}$ & \multirow{2}{*}{\multicolumn{2}{|c|}{ One meal per month }} \\
\hline & Weakfish & & \\
\hline & \begin{tabular}{|l|} 
Bluefish (greater \\
than 14 inches.) \\
\end{tabular} & One meal per year & Do not eat \\
\hline & Striped Bass & \multirow{5}{*}{ One meal per year } & \multirow{5}{*}{ Do not eat } \\
\hline & White perch & & \\
\hline & American eel & & \\
\hline & Channel catfish & & \\
\hline & White catfish & & \\
\hline $\begin{array}{l}\text { DELAWARE BAY } \\
\underline{\text { TRIBUTARIES }}\end{array}$ & American eel & One meal per month & Four meals per year \\
\hline \multicolumn{4}{|c|}{ FISH $\frac{\text { WATERBODY SPECIFIC }}{\text { CONSUMPTION ADVISORIES }}$} \\
\hline \multicolumn{4}{|c|}{ FRESHWATER ADVISORIES } \\
\hline \multirow[b]{2}{*}{ WATERBODY } & \multirow[b]{2}{*}{ SPECIES } & $\begin{array}{l}\text { GENERAL } \\
\text { POPULATION }\end{array}$ & $\begin{array}{l}\text { HIGH-RISK } \\
\text { INDIVIDUAL }\end{array}$ \\
\hline & & $\begin{array}{l}\text { EAT NO MORE } \\
\text { THAN: }\end{array}$ & $\begin{array}{l}\text { EAT NO MORE } \\
\text { THAN: }\end{array}$ \\
\hline $\begin{array}{l}\text { Alycon Lake } \\
\text { (Gloucester Co.) (P) }\end{array}$ & Black Crappie & No restrictions & One meal per month \\
\hline $\begin{array}{l}\text { Assunpink Creek } \\
\text { (Mercer/Monmouth Co.) }\end{array}$ & Largemouth Bass & No restrictions & One meal per week \\
\hline \multirow{2}{*}{$\begin{array}{l}\text { Atlantic City Reservoir - } \\
\text { (Atlantic Co.) (P) } \\
\text { No Fishing Allowed }\end{array}$} & Chain Pickerel & \multirow[b]{2}{*}{ Do not eat } & \multirow[b]{2}{*}{ Do not eat } \\
\hline & \begin{tabular}{|l} 
Largemouth Bass \\
Yellow Perch
\end{tabular} & & \\
\hline \multirow{4}{*}{$\begin{array}{l}\text { Batsto Lake } \\
\text { (Burlington Co.) (P) }\end{array}$} & Chain Pickerel & \multirow{2}{*}{ One meal per week } & \multirow{2}{*}{ Do not eat } \\
\hline & Largemouth Bass & & \\
\hline & Brown Bullhead & \multirow{2}{*}{ No restrictions } & \multirow{2}{*}{ One meal per month } \\
\hline & Yellow Bullhead & & \\
\hline
\end{tabular}

NOTE: To reduce your exposure, eat those fish with the lowest meal restrictions. Do not combine meal restrictions. (For example: If you eat a fish at "one meal per week", then do not eat a different fish at "four meals per year"). 


\begin{tabular}{|c|c|c|c|}
\hline \multicolumn{4}{|c|}{$\begin{array}{l}\text { WATERBODY SPECIFIC } \\
\text { CONSUMPTION ADVISORIES }\end{array}$} \\
\hline \multicolumn{4}{|c|}{ FRESHWATER ADVISORIES } \\
\hline \multirow[b]{2}{*}{ WATERBODY } & \multirow[b]{2}{*}{ SPECIES } & $\begin{array}{l}\text { GENERAL } \\
\text { POPULATION }\end{array}$ & $\begin{array}{l}\text { HIGH-RISK } \\
\text { INDIVIDUAL }\end{array}$ \\
\hline & & $\begin{array}{l}\text { EAT NO MORE } \\
\text { THAN: }\end{array}$ & $\begin{array}{l}\text { EAT NO MORE } \\
\text { THAN: }\end{array}$ \\
\hline \multirow{4}{*}{$\begin{array}{l}\text { Big Timber Creek } \\
\text { (Gloucester Co.) }\end{array}$} & Channel Catfish & \multirow{4}{*}{ No restrictions } & \multirow{3}{*}{ One meal per week } \\
\hline & Largemouth Bass & & \\
\hline & White Catfish & & \\
\hline & Brown Bullhead & & No restrictions \\
\hline \multirow{4}{*}{$\begin{array}{l}\text { Boonton Reservoir } \\
\text { (Morris Co.) }\end{array}$} & Largemouth Bass & \multirow{2}{*}{ Four meals per year } & \multirow{2}{*}{ Do Not Eat } \\
\hline & Smallmouth Bass & & \\
\hline & \begin{tabular}{|l|} 
Rock Bass \\
White
\end{tabular} & $\begin{array}{c}\text { No restriction } \\
\text { One moal ner }\end{array}$ & \multirow{2}{*}{ One meal per month } \\
\hline & \begin{tabular}{|l|} 
White Catfish \\
Brown Bullhead
\end{tabular} & $\begin{array}{c}\text { One meal per week } \\
\text { No restrictions }\end{array}$ & \\
\hline $\begin{array}{l}\text { Bound Brook (Entire length } \\
\text { including New Market Pond \& } \\
\text { Spring Lake) (Somerset Co.) }\end{array}$ & All fish species & Do not eat & Do not eat \\
\hline \multirow{3}{*}{$\begin{array}{l}\text { Branch Brook Park } \\
\text { Newark (Essex Co.) }\end{array}$} & Largemouth Bass & One meal per week & \multirow{2}{*}{ Do not eat } \\
\hline & \begin{tabular}{|l|} 
Common Carp \\
\end{tabular} & One meal per month & \\
\hline & Bluegill & No restrictions & One meal per week \\
\hline \multirow{2}{*}{$\begin{array}{l}\text { Budd Lake } \\
\text { (Morris Co.) }\end{array}$} & Northern Pike & \multirow{2}{*}{ No restrictions } & \multirow{2}{*}{ One meal per week } \\
\hline & White Catfish & & \\
\hline \multirow{2}{*}{$\begin{array}{l}\text { Butterfly Bogs Pond } \\
\text { (Ocean Co.) (P) }\end{array}$} & Chain Pickerel & One meal per week & Do not eat \\
\hline & Brown Bullhead & No restrictions & One meal per week \\
\hline \multirow{3}{*}{$\begin{array}{l}\text { Canistear Reservoir } \\
\text { (Sussex Co.) }\end{array}$} & Chain Pickerel & \multirow{3}{*}{ No restrictions } & \multirow{2}{*}{ One meal per month } \\
\hline & \begin{tabular}{|l|} 
Yellow Perch \\
\end{tabular} & & \\
\hline & \begin{tabular}{|l|} 
Yellow Bullhead \\
Bluegill Sunfish \\
\end{tabular} & & One meal per week \\
\hline \multirow{4}{*}{$\begin{array}{l}\text { Carnegie Lake } \\
\text { (Mercer Co.) }\end{array}$} & Largemouth Bass & One meal per week & Do not eat \\
\hline & \begin{tabular}{|l|} 
Channel Catfish \\
\end{tabular} & \multirow{3}{*}{ No restrictions } & \multirow{2}{*}{ One meal per month } \\
\hline & White Perch & & \\
\hline & Bluegill Sunfish & & No restrictions \\
\hline \multirow{2}{*}{$\begin{array}{l}\text { Cedar Lake } \\
\text { (Cumberland Co.) (P) }\end{array}$} & Chain Pickerel & \multirow{2}{*}{ One meal per week } & \multirow{2}{*}{ Do not eat } \\
\hline & Largemouth Bass & & \\
\hline \multirow{2}{*}{$\begin{array}{l}\text { Clementon Lake } \\
\text { (Camden Co.) (P) }\end{array}$} & Chain Pickerel & \multirow{2}{*}{ One meal per week } & One meal ner month \\
\hline & Largemouth Bass & & \\
\hline Clinton Reservoir & Largemouth Bass & One moal nor weok & Do not eat \\
\hline (Passaic Co.) & Yellow Bullhead & Gile inear per ween & \\
\hline & Rock Bass & ictions & One meal per month \\
\hline & White Sucker & ICtionis & \\
\hline Cooper River, below Evans & \begin{tabular}{|l|} 
Common Carp \\
\end{tabular} & One meal per month & Do not eat \\
\hline Pond (Camden Co.) & Bluegill Sunfish & One meal per week & One meal per month \\
\hline
\end{tabular}

NOTE: To reduce your exposure, eat those fish with the lowest meal restrictions. Do not combine meal restrictions. (For example: If you eat a fish at "one meal per week", then do not eat a different fish at "four meals per year"). 


\begin{tabular}{|c|c|c|c|}
\hline \multicolumn{4}{|c|}{ FISH $\frac{\text { WATERBODY SPECIFIC }}{\text { CONSUMPTION ADVISORIES }}$} \\
\hline \multicolumn{4}{|c|}{ FRESHWATER ADVISORIES } \\
\hline \multirow[b]{2}{*}{ WATERBODY } & \multirow[b]{2}{*}{ SPECIES } & $\begin{array}{c}\text { GENERAL } \\
\text { POPULATION }\end{array}$ & $\begin{array}{l}\text { HIGH-RISK } \\
\text { INDIVIDUAL }\end{array}$ \\
\hline & & $\begin{array}{l}\text { EAT NO MORE } \\
\text { THAN: }\end{array}$ & $\begin{array}{l}\text { EAT NO MORE } \\
\text { THAN: }\end{array}$ \\
\hline $\begin{array}{l}\text { Cooper River, Hopkins Pond } \\
\text { (Camden Co.) }\end{array}$ & Brown Bullhead & One meal per month & Four meals per year \\
\hline \multirow{3}{*}{$\begin{array}{l}\text { Cooper River Lake } \\
\text { (Camden Co.) }\end{array}$} & Largemouth Bass & \multirow{2}{*}{ Four meals per year } & \multirow{2}{*}{ Do not eat } \\
\hline & Common Carp & & \\
\hline & $\begin{array}{l}\text { Brown Bullhead } \\
\text { Bluegill Sunfish }\end{array}$ & One meal per week & One meal per month \\
\hline $\begin{array}{l}\text { Cranbury Lake } \\
\text { (Sussex Co) }\end{array}$ & Hybrid Striped Bass & One meal per week & One meal per month \\
\hline \multirow{2}{*}{$\begin{array}{l}\text { Crater Lake } \\
\text { (Sussex Co.) }\end{array}$} & \begin{tabular}{|l|} 
Yellow Perch \\
\end{tabular} & \multirow{2}{*}{ One meal per week } & Do not eat \\
\hline & Brown Bullhead & & One meal per month \\
\hline \multirow{2}{*}{$\begin{array}{l}\text { Crosswicks Creek } \\
\text { (Mercer Co.) }\end{array}$} & Largemouth Bass & \multirow{2}{*}{ No restrictions } & \multirow{2}{*}{ One meal per week } \\
\hline & White Catfish & & \\
\hline \multirow{3}{*}{$\begin{array}{l}\text { Crystal Lake } \\
\text { (Burlington Co.) }\end{array}$} & Largemouth Bass & \multirow{3}{*}{ No restrictions } & One meal per month \\
\hline & Black Crappie & & One meal per week \\
\hline & Brown Bullhead & & No restrictions \\
\hline \multirow{2}{*}{$\begin{array}{l}\text { DeVoe Lake } \\
\text { (Middlesex Co.) }\end{array}$} & Chain Pickerel & \multirow{2}{*}{ No restrictions } & \multirow{2}{*}{ One meal per month } \\
\hline & Largemouth Bass & & \\
\hline $\begin{array}{l}\text { Delaware \& Raritan Canal @ } \\
\text { Bound Brook (Somerset Co.) }\end{array}$ & Channel Catfish & One meal per week & Do not eat \\
\hline \multirow{4}{*}{$\begin{array}{l}\text { Delaware River } \\
\text { Upstream of Watergap } \\
\text { (Warren/Sussex Co) }\end{array}$} & Channel Catfish & \multirow{2}{*}{ No restrictions } & \multirow{2}{*}{ One meal per month } \\
\hline & Muskellunge & & \\
\hline & Smallmouth Bass & \multicolumn{2}{|c|}{ One meal per week } \\
\hline & White Sucker & \multicolumn{2}{|c|}{ One meal per month } \\
\hline \multirow{3}{*}{$\begin{array}{l}\text { Delaware River } \\
\text { Watergap to Phillipsburg } \\
\text { (Warren Co.) }\end{array}$} & \begin{tabular}{|l} 
White Catfish \\
\end{tabular} & One meal per week & Do not eat \\
\hline & $\begin{array}{l}\text { Channel Catfish } \\
\text { Smallmouth Bass }\end{array}$ & \multirow[t]{2}{*}{ No restrictions } & One meal per month \\
\hline & Walleye & & One meal per week \\
\hline \multirow{6}{*}{$\begin{array}{l}\text { Delaware River } \\
\text { Phillipsburg to Trenton } \\
\text { (Hunterdon/Mercer Co.) }\end{array}$} & Channel Catfish & Four meals per year & Do not eat \\
\hline & White Sucker & \multicolumn{2}{|c|}{ One meal per month } \\
\hline & Largemouth Bass & No restrictions & One meal per month \\
\hline & Smallmouth Bass & One meal per week & One meal per month \\
\hline & American Eel & One meal per month & \multirow{2}{*}{ Do not eat } \\
\hline & Striped Bass & Four meals per year & \\
\hline $\begin{array}{l}\text { Double Trouble Lake } \\
\text { (Ocean Co.) (P) }\end{array}$ & Yellow Bullhead & One meal per month & Do not eat \\
\hline
\end{tabular}

NOTE: To reduce your exposure, eat those fish with the lowest meal restrictions. Do not combine meal restrictions. (For example: If you eat a fish at "one meal per week", then do not eat a different fish at "four meals per year"). 


\section{WATERBODY SPECIFIC FISH CONSUMPTION ADVISORIES}

FRESHWATER ADVISORIES

\begin{tabular}{|c|c|c|c|}
\hline \multirow[b]{2}{*}{ WATERBODY } & \multirow[b]{2}{*}{ SPECIES } & $\begin{array}{c}\text { GENERAL } \\
\text { POPULATION }\end{array}$ & $\begin{array}{l}\text { HIGH-RISK } \\
\text { INDIVIDUAL }\end{array}$ \\
\hline & & $\begin{array}{c}\text { EAT NO MORE } \\
\text { THAN: }\end{array}$ & $\begin{array}{l}\text { EAT NO MORE } \\
\text { THAN: }\end{array}$ \\
\hline \multirow{3}{*}{$\begin{array}{l}\text { East Creek Lake } \\
\text { (Cape May Co.) (P) }\end{array}$} & Brown Bullhead & \multirow{3}{*}{ One meal per month } & \multirow{3}{*}{ Do not eat } \\
\hline & Yellow Bullhead & & \\
\hline & Yellow Perch & & \\
\hline \multirow{4}{*}{$\begin{array}{l}\text { Echo Lake Reservoir } \\
\text { (Passaic Co.) }\end{array}$} & Largemouth Bass & \multirow{4}{*}{ No restrictions } & \multirow{2}{*}{ One meal per month } \\
\hline & Chain Pickerel & & \\
\hline & Bluegill Sunfish & & \multirow{2}{*}{ One meal per week } \\
\hline & Yellow Bullhead & & \\
\hline Evans Pond (Camden Co.) & Brown Bullhead & One meal per week & One meal per month \\
\hline \multirow{2}{*}{$\begin{array}{l}\text { Green Turtle Lake } \\
\text { (Passaic Co.) }\end{array}$} & Chain Pickerel & \multirow{2}{*}{ No restrictions } & \multirow{2}{*}{ One meal per week } \\
\hline & Yellow Perch & & \\
\hline \multirow{5}{*}{$\begin{array}{l}\text { Greenwood Lake } \\
\text { (Passaic Co.) }\end{array}$} & Largemouth Bass & \multirow{5}{*}{ No restrictions } & \multirow{2}{*}{ One meal per month } \\
\hline & Walleye & & \\
\hline & White Perch & & No restrictions \\
\hline & Bluegill Sunfish & & \multirow{2}{*}{ One meal per week } \\
\hline & Yellow Bullhead & & \\
\hline \multirow{2}{*}{$\begin{array}{l}\text { Grovers Mill Pond } \\
\text { (Mercer Co.) }\end{array}$} & Brown Bullhead & One meal per week & One meal per month \\
\hline & Chain Pickerel & No restrictions & One meal per week \\
\hline \multirow{2}{*}{$\begin{array}{l}\text { Hainesville Pond } \\
\text { (Sussex Co.) }\end{array}$} & Largemouth Bass & \multirow{2}{*}{ No restrictions } & One meal per month \\
\hline & Chain Pickerel & & One meal per week \\
\hline \multirow{2}{*}{$\begin{array}{l}\text { Harrisville Lake } \\
\text { (Burlington Co.) (P) }\end{array}$} & Mud Sunfish & \multirow{2}{*}{ One meal per month } & \multirow{2}{*}{ Do not eat } \\
\hline & Yellow Bullhead & & \\
\hline \multirow{2}{*}{$\begin{array}{l}\text { Lake Carasaljo } \\
\text { (Ocean Co.) (P) }\end{array}$} & Largemouth Bass & \multirow{2}{*}{ One meal per week } & Do not eat \\
\hline & Chain Pickerel & & One meal per month \\
\hline $\begin{array}{l}\text { Lake Hopatcong } \\
\text { (Morris/Sussex Co.) }\end{array}$ & Largemouth Bass & No restrictions & One meal per month \\
\hline \multirow{3}{*}{$\begin{array}{l}\text { Lake Nummy } \\
\text { (Cape May Co.) (P) }\end{array}$} & Chain Pickerel & \multirow{2}{*}{ One meal per week } & \multirow{2}{*}{ Do not eat } \\
\hline & Yellow Perch & & \\
\hline & Yellow Bullhead & No restrictions & One meal per month \\
\hline \multirow{5}{*}{$\begin{array}{l}\text { Lake Tappan } \\
\text { (Bergen Co.) }\end{array}$} & Smallmouth Bass & \multirow{5}{*}{ No restriction } & One meal nor month \\
\hline & Largemouth Bass & & \\
\hline & Bluegill Sunfish & & No restriction \\
\hline & Yellow Bullhead & & meal ner week \\
\hline & Common Carp & & Ulle medr per week \\
\hline $\begin{array}{l}\text { Lenape Lake } \\
\text { (Atlantic Co.) (P) }\end{array}$ & Chain Pickerel & One meal per week & Do not eat \\
\hline Linden Lake (Camden Co) (P) & Largemouth Bass & No restrictions & One meal per month \\
\hline
\end{tabular}

NOTE: To reduce your exposure, eat those fish with the lowest meal restrictions. Do not combine meal restrictions. (For example: If you eat a fish at "one meal per week", then do not eat a different fish at "four meals per year"). 


\begin{tabular}{|c|c|c|c|}
\hline${ \hline\multicolumn{4}{|c|}{}}$
\end{tabular}$} \\
{\hline \multicolumn{4}{|c|}{\text { FRESHWATER ADVISORIES }}\text { FRESHWATER ADVISORIES }} \\
{\hline \multirow[b]{2}{*}{\text { WATERBODY }}\text { WATERBODY }} &{\multirow[b]{2}{*}{\text { SPECIES }}\text { SPECIES }} &{\begin{array}{l}\text { GENERAL } \\
\text { POPULATION }\end{array}} &{\begin{array}{l}\text { HIGH-RISK } \\
\text { INDIVIDUAL }\end{array}} \\
{\hline} &{ } &{\begin{array}{l}\text { EAT NO MORE } \\
\text { THAN: }\end{array}} &{\begin{array}{l}\text { EAT NO MORE } \\
\text { THAN: }\end{array}} \\
{\hline \begin{array}{l}\text { Little Timber Creek } \\
\text { (Camden Co.) }\end{array}} &{\text { Brown Bullhead }} &{\text { No restrictions }} &{\text { No restrictions }} \\
{\hline \multirow{3}{*}{\begin{array}{l}\text { Manasquan Reservoir } \\
\text { (Monmouth Co.) }\end{array}}\begin{array} { l } { \text { Manasquan Reservoir } } \\
{ \text { (Monmouth Co.) } } \end{array}} &{\text { Largemouth Bass }} &{\text { One meal per month }} &{\text { Do not eat }} \\
{\hline} &{\text { Black Crappie }} &{\text { One meal per week }} &{\multirow{2}{*}{\text { One meal per month }}\text { One meal per month }} \\
{\hline} &{\begin{array}{l}\text { Chain Pickerel } \\
\text { Yellow Perch }\end{array}} &{\text { No restrictions }} &{ } \\
{\hline \multirow{4}{*}{\begin{array}{l}\text { Maskells Mill Lake } \\
\text { (Salem Co.) (P) }\end{array}}\begin{array} { l } { \text { Maskells Mill Lake } } \\
{ \text { (Salem Co.) (P) } } \end{array}} &{\text { Brown Bullhead }} &{\multirow{3}{*}{\text { One meal per week }}\text { One meal per week }} &{\multirow{4}{*}{\text { One meal per month }}\text { One meal per month }} \\
{\hline} &{\text { Chain Pickerel }} &{ } &{ } \\
{\hline} &{\text { Largemouth Bass }} &{ } &{ } \\
{\hline} &{\text { Black Crappie }} &{\text { No restrictions }} &{ } \\
{\hline \multirow{6}{*}{\begin{array}{l}\text { Merrill Creek Reservoir } \\
\text { (Warren Co.) }\end{array}}\begin{array} { l } { \text { Merrill Creek Reservoir } } \\
{ \text { (Warren Co.) } } \end{array}} &{\text { Largemouth Bass }} &{\text { One meal per month }} &{\multirow[b]{2}{*}{\text { Do not eat }}\text { Do not eat }} \\
{\hline} &{\begin{array}{l}\text { Smallmouth Bass } \\
\text { Lake Trout }\end{array}} &{\text { One meal per week }} &{ } \\
{\hline} &{\text { Yellow Perch }} &{\multirow{4}{*}{\text { No restrictions }}\text { No restrictions }} &{\text { One meal per month }} \\
{\hline} &{\text { Black Crappie }} &{ } &{ } \\
{\hline} &{\text { Bluegill Sunfish }} &{ } &{\text { One meal per week }} \\
{\hline} &{\text { Brown Bullhead }} &{ } &{ } \\
{\hline \multirow{2}{*}{\begin{array}{l}\text { Mirror Lake } \\
\text { (Burlington Co.) (P) }\end{array}}\begin{array} { l } { \text { Mirror Lake } } \\
{ \text { (Burlington Co.) (P) } } \end{array}} &{\text { Largemouth Bass }} &{\text { One meal per week }} &{\text { One meal per month }} \\
{\hline} &{\text { Brown Bullhead }} &{\text { No restrictions }} &{\text { One meal per week }} \\
{\hline \multirow{5}{*}{\begin{array}{l}\text { Monksville Reservoir } \\
\text { (Passaic Co.) }\end{array}}\begin{array} { l } { \text { Monksville Reservoir } } \\
{ \text { (Passaic Co.) } } \end{array}} &{\text { Smallmouth Bass }} &{\multirow{3}{*}{\text { No restrictions }}\text { No restrictions }} &{\text { One meal per month }} \\
{\hline} &{\text { Yellow Bullhead }} &{ } &{\multirow[b]{2}{*}{\text { One meal per week }}\text { One meal per week }} \\
{\hline} &{\begin{array}{l}\text { Bluegill Sunfish } \\
\text { Yellow Perch }\end{array}} &{ } &{ } \\
{\hline} &{\text { White Perch }} &{\multirow{2}{*}{\text { One meal per week }}\text { One meal per week }} &{\multirow{2}{*}{\text { Do not eat }}\text { Do not eat }} \\
{\hline} &{\text { Walleye }} &{ } &{ } \\
{\hline \begin{array}{l}\text { Mountain Lake. } \\
\text { (Warren Co) }\end{array}} &{\text { Largemouth Bass }} &{\text { One meal per week }} &{\text { Do not eat }} \\
{\hline \multirow{3}{*}{\begin{array}{l}\text { Mullica River } \\
\text { (Burlington/Atlantic Co) (P) }\end{array}}\begin{array} { l } { \text { Mullica River } } \\
{ \text { (Burlington/Atlantic Co) (P) } } \end{array}} &{\text { Brown Bullhead }} &{\multirow{2}{*}{\text { One meal per week }}\text { One meal per week }} &{\multirow{3}{*}{\text { One meal per month }}\text { One meal per month }} \\
{\hline} &{\text { White Perch }} &{ } &{ } \\
{\hline} &{\text { White Catfish }} &{\text { No restrictions }} &{ } \\
{\hline \multirow{5}{*}{\begin{array}{l}\text { New Brooklyn Lake } \\
\text { (Camden Co.) (P) }\end{array}}\begin{array} { l } { \text { New Brooklyn Lake } } \\
{ \text { (Camden Co.) (P) } } \end{array}} &{\text { Chain Pickerel }} &{\multirow{2}{*}{\text { One meal per week }}\text { One meal per week }} &{\text { Do not eat }} \\
{\hline} &{\text { Largemouth Bass }} &{ } &{\multirow{3}{*}{\text { One meal per month }}\text { One meal per month }} \\
{\hline} &{\text { Pumpkinseed Sunfish }} &{\multirow{3}{*}{\text { No restrictions }}\text { No restrictions }} &{ } \\
{\hline} &{\text { Black Crappie }} &{ } &{ } \\
{\hline} &{\text { Yellow Bullhead }} &{ } &{\text { One meal per week }} \\
{\hline \begin{array}{l}\text { Newton Creek, North } \\
\text { (Camden Co.) }\end{array}} &{\text { Brown Bullhead }} &{\text { No restrictions }} &{\text { No restrictions }} \\
$\hline$\end{array}$

NOTE: To reduce your exposure, eat those fish with the lowest meal restrictions. Do not combine meal restrictions. (For example: If you eat a fish at "one meal per week", then do not eat a different fish at "four meals per year"). 


\begin{tabular}{|c|c|c|c|}
\hline \multicolumn{4}{|c|}{$\begin{array}{ll}\text { FISH } & \frac{\text { WTERBODY SPECIFIC }}{\text { CONSUMPTION ADVISORIES }} \\
\end{array}$} \\
\hline \multicolumn{4}{|c|}{ FRESHWATER ADVISORIES } \\
\hline \multirow[b]{2}{*}{ WATERBODY } & \multirow[b]{2}{*}{ SPECIES } & $\begin{array}{l}\text { GENERAL } \\
\text { POPULATION }\end{array}$ & $\begin{array}{l}\text { HIGH-RISK } \\
\text { INDIVIDUAL }\end{array}$ \\
\hline & & $\begin{array}{l}\text { EAT NO MORE } \\
\text { THAN: }\end{array}$ & $\begin{array}{l}\text { EAT NO MORE } \\
\text { THAN: }\end{array}$ \\
\hline $\begin{array}{l}\text { Newton Creek, South } \\
\text { (Camden Co.) }\end{array}$ & Largemouth Bass & One meal per month & Do not eat \\
\hline \multirow{4}{*}{$\begin{array}{l}\text { Newton Lake } \\
\text { (Camden Co.) }\end{array}$} & \begin{tabular}{|l|} 
Bluegill Sunfish \\
\end{tabular} & \multirow{2}{*}{ One meal per week } & \multirow{2}{*}{ One meal per montl } \\
\hline & \begin{tabular}{|l} 
Brown Bullhead \\
\end{tabular} & & \\
\hline & \begin{tabular}{|l} 
Largemouth Bass \\
\end{tabular} & \multirow{2}{*}{ One meal per month } & Four meals per year \\
\hline & Common Carp & & Do not eat \\
\hline \multirow{3}{*}{$\begin{array}{l}\text { Oak Ridge Reservoir } \\
\text { (Passaic Co.) }\end{array}$} & \begin{tabular}{|l} 
Largemouth Bass \\
\end{tabular} & One meal per week & Do not eat \\
\hline & Chain Pickerel & \multirow{2}{*}{ No restrictions } & One meal per month \\
\hline & Brown Bullhead & & No restrictions \\
\hline \multirow{4}{*}{$\begin{array}{l}\text { Oradell Reservoir } \\
\text { (Bergen Co.) }\end{array}$} & Largemouth Bass & \multirow{4}{*}{ No restriction } & One meal per month \\
\hline & Bluegill Sunfish & & \\
\hline & Yellow Bullhead & & No restrictions \\
\hline & Common Carp & & \\
\hline \multirow{3}{*}{$\begin{array}{l}\text { Overpeck Creek (Branch of } \\
\text { the Hackensack River; } \\
\text { Bergen Co). }\end{array}$} & Largemouth Bass & \multirow{3}{*}{ Four meals per year } & \multirow{3}{*}{ Do not eat } \\
\hline & \begin{tabular}{|l|} 
Common Carp \\
\end{tabular} & & \\
\hline & American Eel & & \\
\hline \multirow{5}{*}{$\begin{array}{l}\text { Passaic River: } \\
\text { Rt. } 280 \text { to confluence of } \\
\text { Pompton R. to Two Bridges } \\
\text { (Morris/Essex/Passaic Co.) }\end{array}$} & Redbreast Sunfish & \multirow{2}{*}{ One meal per week } & One meal per montl \\
\hline & \begin{tabular}{|l|} 
Northern Pike \\
Common Carp \\
\end{tabular} & & Do not eat \\
\hline & Black Crappie & \multirow{3}{*}{ No restrictions } & One meal per month \\
\hline & \begin{tabular}{|l} 
Yellow Bullhead \\
\end{tabular} & & \multirow{2}{*}{ One meal per week } \\
\hline & \begin{tabular}{|l|} 
Pumpkinseed Sunfish \\
\end{tabular} & & \\
\hline \multirow{6}{*}{$\begin{array}{l}\text { Passaic River: } \\
\text { Elmwood Park to Dundee } \\
\text { Lake } \\
\text { (Passaic/Bergen Co.) }\end{array}$} & Largemouth Bass & \multirow{3}{*}{ One meal per week } & \multirow{2}{*}{ One meal per month } \\
\hline & Yellow Bullhead & & \\
\hline & \begin{tabular}{|l|} 
Brown Bullhead \\
\end{tabular} & & Four meals per year \\
\hline & \begin{tabular}{|l} 
Redbreast Sunfish \\
Common Carp
\end{tabular} & & \\
\hline & Bluegill Sunfish & One meal per month & Do not eat \\
\hline & \begin{tabular}{|l|} 
American Eel \\
\end{tabular} & & \\
\hline Pennsauken Creek, Forked & Common Carp & Four meals per year & Do not \\
\hline Landing (Camden Co.) & Largemouth Bass & & Do not eat \\
\hline & \begin{tabular}{|l|} 
Pumpkinseed Sunfish \\
\end{tabular} & One meal per month & Four meals per year \\
\hline & \begin{tabular}{|l|} 
White Catfish \\
\end{tabular} & & One meal per year \\
\hline Pompton Lake & Largemouth Bass & One meal per week & \\
\hline (Passaic Co.) & Common Carp & One meal per month & Do not eat \\
\hline
\end{tabular}

NOTE: To reduce your exposure, eat those fish with the lowest meal restrictions. Do not combine meal restrictions. (For example: If you eat a fish at "one meal per week", then do not eat a different fish at "four meals per year"). 


\section{WATERBODY SPECIFIC FISH CONSUMPTION ADVISORIES}

\begin{tabular}{|c|c|c|c|}
\hline \multicolumn{4}{|c|}{ FRESHWATER ADVISORIES } \\
\hline \multirow[b]{2}{*}{ WATERBODY } & \multirow[b]{2}{*}{ SPECIES } & $\begin{array}{c}\text { GENERAL } \\
\text { POPULATION }\end{array}$ & $\begin{array}{l}\text { HIGH-RISK } \\
\text { INDIVIDUAL }\end{array}$ \\
\hline & & $\begin{array}{l}\text { EAT NO MORE } \\
\text { THAN: }\end{array}$ & $\begin{array}{l}\text { EAT NO MORE } \\
\text { THAN: }\end{array}$ \\
\hline \multirow{7}{*}{$\begin{array}{l}\text { Pompton River at Lincoln } \\
\text { Park } \\
\text { (Passaic/Morris Co.) }\end{array}$} & Largemouth Bass & \multirow{4}{*}{ One meal per week } & One meal per month \\
\hline & Northern Pike & & \multirow{2}{*}{ Do not eat } \\
\hline & Rock Bass & & \\
\hline & Redbreast Sunfish & & Four meals per year \\
\hline & Black Crappie & \multirow{2}{*}{ No restrictions } & \multirow{2}{*}{ One meal per month } \\
\hline & Yellow Perch & & \\
\hline & Common Carp & Four meals per year & Do not eat \\
\hline \multirow{7}{*}{$\begin{array}{l}\text { Ramapo River } \\
\text { @ Pompton Feeder } \\
\text { (Morris Co.) } \\
\text { Site formerly listed as } \\
\text { Pompton River @ } \\
\text { Pequannock R. } \\
\text { (Passaic/Morris Co.) }\end{array}$} & Largemouth Bass & \multirow{7}{*}{ One meal per week } & \multirow{5}{*}{ Do not eat } \\
\hline & Smallmouth Bass & & \\
\hline & Yellow Bullhead & & \\
\hline & Rock Bass & & \\
\hline & Pumpkinseed Sunfish & & \\
\hline & Redbreast Sunfish & & \multirow{2}{*}{ One meal per month } \\
\hline & Black Crappie & & \\
\hline \multirow{4}{*}{$\begin{array}{l}\text { Raritan River at Neshanic } \\
\text { Station } \\
\text { (Somerset Co.) }\end{array}$} & Largemouth Bass & \multirow{4}{*}{ No restrictions } & \multirow{4}{*}{ One meal per week } \\
\hline & Smallmouth Bass & & \\
\hline & Redbreast Sunfish & & \\
\hline & Rock Bass & & \\
\hline \multirow{2}{*}{$\begin{array}{l}\text { Raritan River at Millstone } \\
\text { River (Somerset Co.) }\end{array}$} & Channel Catfish & \multirow{2}{*}{ No restrictions } & One meal per week \\
\hline & Brown Bullhead & & No restrictions \\
\hline \multirow{5}{*}{$\begin{array}{l}\text { Rockaway River @ } \\
\text { Powerville } \\
\text { (Morris Co.) }\end{array}$} & Largemouth Bass & One meal per week & Do not eat \\
\hline & Yellow Bullhead & \multirow{4}{*}{ No restrictions } & \multirow{2}{*}{ One meal per week } \\
\hline & Bluegill Sunfish & & \\
\hline & Rock Bass & & \multirow{2}{*}{ One meal per month } \\
\hline & Chain Pickerel & & \\
\hline \multirow{3}{*}{$\begin{array}{l}\text { Rockaway River at } \\
\text { Whippany } \\
\text { (Morris Co.) }\end{array}$} & Largemouth Bass & One meal per week & Do not eat \\
\hline & Black Crappie & \multirow{2}{*}{ No restrictions } & One meal per month \\
\hline & Bluegill Sunfish & & One meal per week \\
\hline \multirow{2}{*}{$\begin{array}{l}\text { Round Valley Reservoir } \\
\text { (Hunterdon Co.) }\end{array}$} & Largemouth Bass & \multirow{2}{*}{ No restrictions } & One meal per month \\
\hline & Lake Trout & & One meal per week \\
\hline \multirow{2}{*}{$\begin{array}{l}\text { Saw Mill Lake } \\
\text { (Sussex Co.) }\end{array}$} & Northern Pike & \multirow{2}{*}{ No restrictions } & One meal per month \\
\hline & Brown Bullhead & & No restrictions \\
\hline $\begin{array}{l}\text { Shadow Lake } \\
\text { (Monmouth Co.) }\end{array}$ & Largemouth Bass & No restrictions & One meal per week \\
\hline
\end{tabular}

NOTE: To reduce your exposure, eat those fish with the lowest meal restrictions. Do not combine meal restrictions. (For example: If you eat a fish at "one meal per week", then do not eat a different fish at "four meals per year"). 


\section{WATERBODY SPECIFIC FISH CONSUMPTION ADVISORIES}

\begin{tabular}{|c|c|c|c|}
\hline \multicolumn{4}{|c|}{ FRESHWATER ADVISORIES } \\
\hline \multirow[b]{2}{*}{ WATERBODY } & \multirow[b]{2}{*}{ SPECIES } & $\begin{array}{l}\text { GENERAL } \\
\text { POPULATION }\end{array}$ & $\begin{array}{l}\text { HIGH-RISK } \\
\text { INDIVIDUAL }\end{array}$ \\
\hline & & $\begin{array}{l}\text { EAT NO MORE } \\
\text { THAN: }\end{array}$ & $\begin{array}{l}\text { EAT NO MORE } \\
\text { THAN: }\end{array}$ \\
\hline \multirow{2}{*}{$\begin{array}{l}\text { Shepherd Lake (a.k.a. } \\
\text { Sheppards Lake) } \\
\text { (Passaic Co.) }\end{array}$} & Largemouth Bass & One meal per week & Do not eat \\
\hline & Rock Bass & No restrictions & One meal per week \\
\hline \multirow{3}{*}{\begin{tabular}{|l} 
Speedwell Lake \\
(Morris Co.)
\end{tabular}} & Bluegill Sunfish & \multirow{3}{*}{ No restrictions } & \multirow{3}{*}{ One meal per week } \\
\hline & Chain Pickerel & & \\
\hline & Common carp & & \\
\hline \multirow{3}{*}{\begin{tabular}{|l}
$\begin{array}{l}\text { Splitrock Reservoir } \\
\text { (Morris Co.) }\end{array}$ \\
\end{tabular}} & Chain Pickerel & \multirow{3}{*}{ No restrictions } & One meal per month \\
\hline & Yellow Perch & & \\
\hline & \begin{tabular}{|l|} 
Bluegill Sunfish \\
Brown Bullhead \\
\end{tabular} & & $\begin{array}{l}\text { One meal per week } \\
\text { No restrictions }\end{array}$ \\
\hline \begin{tabular}{|l} 
Spring Lake \\
(Monmouth Co.) (P)
\end{tabular} & Largemouth Bass & One meal per week & Do not eat \\
\hline \multirow{2}{*}{$\begin{array}{l}\text { Spruce Run Reservoir } \\
\text { (Hunterdon Co.) }\end{array}$} & \begin{tabular}{|l|} 
Northern Pike \\
\end{tabular} & One meal per week & \multirow{2}{*}{ One meal per month } \\
\hline & Hybrid Striped Bass & No restrictions & \\
\hline $\begin{array}{l}\text { Stafford Forge Main Line } \\
\text { (Ocean Co.) (P) }\end{array}$ & Chain Pickerel & One meal per week & Do not eat \\
\hline \begin{tabular}{|l|} 
Steenykill Lake \\
(Sussex Co.)
\end{tabular} & Largemouth Bass & No restrictions & One meal per week \\
\hline \multirow{4}{*}{$\begin{array}{l}\text { Stewart Lake } \\
\text { (Camden Co.) }\end{array}$} & Largemouth Bass & Four meals per year & Four meals per year \\
\hline & Bluegill Sunfish & \multirow{2}{*}{ One meal per week } & One meal per month \\
\hline & Brown Bullhead & & \multirow{2}{*}{ Do not eat } \\
\hline & \begin{tabular}{|l|} 
Common Carp \\
\end{tabular} & One meal per month & \\
\hline \multirow{4}{*}{$\begin{array}{l}\text { Strawbridge Lake } \\
\text { (Burlington Co.) }\end{array}$} & Largemouth Bass & \multirow{2}{*}{ One meal per month } & \multirow{2}{*}{ One meal per year } \\
\hline & Bluegill Sunfish & & \\
\hline & Common Carp & Four meals per year & Do not eat \\
\hline & Brown Bullhead & One meal per week & Four meals per year \\
\hline \begin{tabular}{|l|} 
Sunset Lake \\
(Cumberland Co.) (P)
\end{tabular} & Largemouth Bass & One meal per week & One meal per month \\
\hline \multirow{2}{*}{$\begin{array}{l}\text { Swartswood Lake } \\
\text { (Sussex Co.) }\end{array}$} & Smallmouth Bass & \multirow{2}{*}{ No restrictions } & One meal per month \\
\hline & \begin{tabular}{|l|} 
Chain Pickerel \\
\end{tabular} & & One meal per week \\
\hline \begin{tabular}{|l|} 
Union Lake \\
(Cumberland Co.) (P)
\end{tabular} & White Perch & One meal per week & Do not eat \\
\hline \multirow{2}{*}{\begin{tabular}{|l} 
Wading River \\
(Burlington Co.) (P)
\end{tabular}} & Yellow Bullhead & One meal per month & \multirow[b]{2}{*}{ Do not eat } \\
\hline & \begin{tabular}{|l|} 
Chain Pickerel \\
White Catfish
\end{tabular} & One meal per week & \\
\hline
\end{tabular}

NOTE: To reduce your exposure, eat those fish with the lowest meal restrictions. Do not combine meal restrictions. (For example: If you eat a fish at "one meal per week", then do not eat a different fish at "four meals per year"). 


\section{WATERBODY SPECIFIC FISH CONSUMPTION ADVISORIES}

\begin{tabular}{|c|c|c|c|}
\hline \multicolumn{4}{|c|}{ FRESHWATER ADVISORIES } \\
\hline \multirow[b]{2}{*}{ WATERBODY } & \multirow[b]{2}{*}{ SPECIES } & $\begin{array}{c}\text { GENERAL } \\
\text { POPULATION }\end{array}$ & $\begin{array}{c}\text { HIGH-RISK } \\
\text { INDIVIDUAL }\end{array}$ \\
\hline & & $\begin{array}{l}\text { EAT NO MORE } \\
\text { THAN: }\end{array}$ & $\begin{array}{l}\text { EAT NO MORE } \\
\text { THAN: }\end{array}$ \\
\hline \multirow[t]{4}{*}{$\begin{array}{l}\text { Wanaque Reservoir } \\
\text { (Passaic Co.) }\end{array}$} & \begin{tabular}{|l|} 
Largemouth Bass \\
White Perch
\end{tabular} & One meal per week & Do not eat \\
\hline & \begin{tabular}{|l} 
White Catfish \\
\end{tabular} & \multirow{3}{*}{ No restrictions } & One meal per month \\
\hline & Brown Bullhead & & No restrictions \\
\hline & Yellow Bullhead & & One meal per week \\
\hline \multirow{3}{*}{$\begin{array}{l}\text { Wawayanda Lake } \\
\text { (Sussex Co.) }\end{array}$} & Largemouth Bass & \multirow{2}{*}{ One meal per week } & Do not eat \\
\hline & Yellow Bullhead & & \multirow{2}{*}{ One meal per month } \\
\hline & Chain Pickerel & No restriction & \\
\hline \multirow{5}{*}{$\begin{array}{l}\text { Weequahic Lake } \\
\text { (Essex Co.) }\end{array}$} & Largemouth Bass & \multirow{2}{*}{ One meal per month } & One meal per month \\
\hline & Common Carp & & Do not eat \\
\hline & Bluegill & One meal per week & \multirow{2}{*}{ One meal per week } \\
\hline & White Perch & \multirow{2}{*}{ No restrictions } & \\
\hline & Brown Bullhead & & No restrictions \\
\hline $\begin{array}{l}\text { Whitesbog Pond } \\
\text { (Ocean Co.) (P) }\end{array}$ & Chain Pickerel & One meal per week & Do not eat \\
\hline $\begin{array}{l}\text { Willow Grove Lake } \\
\text { (Cumberland Co.) (P) }\end{array}$ & Brown Bullhead & No restrictions & One meal per month \\
\hline \multirow{4}{*}{$\begin{array}{l}\text { Wilson Lake } \\
\text { (Gloucester Co.) (P) }\end{array}$} & Largemouth Bass & One meal per week & \multirow{4}{*}{ Do not eat } \\
\hline & Yellow Perch & \multirow{3}{*}{ One meal per month } & \\
\hline & Chain Pickerel & & \\
\hline & Pumpkinseed Sunfish & & \\
\hline \multirow{2}{*}{$\begin{array}{l}\text { Woodstown Memorial Lake } \\
\text { (Salem Co.) }\end{array}$} & Black Crappie & \multirow{2}{*}{ No restrictions } & \multirow{2}{*}{ One meal per month } \\
\hline & Largemouth Bass & & \\
\hline
\end{tabular}

(1)High-risk individuals include infants, children, pregnant women, nursing mothers and women of childbearing age.

(2)One meal is defined as an eight-ounce serving

(3)Eat only the fillet portions of the fish. Use proper trimming techniques to remove fat, and cooking methods that allow juices to drain from the fish (e.g., baking, broiling, frying, grilling, and steaming). See text for full description.

(4)Sunfish includes bluegill, pumpkinseed, and redbreast sunfish.

(5) No harvest means no taking or attempting to take any blue crabs from these waters.

Note: Not all species were found or analyzed in all water bodies, or inadequate data were available to list some species.

(P) = Pinelands Area

* Selling any of these species from designated water bodies is prohibited in New Jersey. 